Research Article

\title{
Hatha Yoga Improves Psychophysiological Responses of College Students in Both Indoor and Outdoor Environments
}

\author{
Kelsey Madison Dietrich, Maria Gabriela Bidart *
}

Bowling Green State University, Life Sciences Building, Bowling Green, OH, USA; E-Mails: dietkel@bgsu.edu; gbidart@bgsu.edu

* Correspondence: Maria Gabriela Bidart; E-Mail: gbidart@bgsu.edu

Academic Editor: Peta Stapleton

Special Issue: Mind-Body Approaches that are Revolutionizing the Health Field

OBM Integrative and Complementary Medicine 2021, volume 6 , issue 4

doi:10.21926/obm.icm.2104046
Received: July 15, 2021

Accepted: October 29, 2021

Published: November 12, 2021

\begin{abstract}
College students experience increased stress levels that could predispose them to develop mental and physical health conditions throughout adulthood. Yoga is an ancient mind-body practice including breath techniques, body exercises, and meditation that may be a useful strategy for enhancing college student health. Likewise, exposure to nature has been shown to have beneficial impacts on human health. This study investigates physiological and psychological responses of college students to yoga practiced in outdoor versus indoor environments. Fifty-eight college females between the ages of 18-28 were randomly assigned to complete a 40-minute Hatha yoga session either indoors (empty classroom) or outdoors (green park) at a mid-sized public university. Heart rate, respiration rate, perfusion index, and positive and negative affect states were recorded pre/post yoga. Heart rate, respiration rate, and negative affect significantly improved after the yoga sessions in both indoor and outdoor environments. Baseline self-reported negative affect was significantly lower in the outdoor group compared to the indoor group. There were no significant interaction effects of yoga and environment. This study suggests that one 40-minute-session of Hatha yoga may be a useful method to alleviate acute signs of physiological stress and decrease negative affect for college females in both indoor and outdoor environments. Further research about potential
\end{abstract}

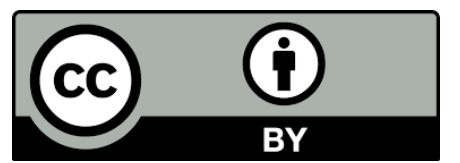

(C) 2021 by the author. This is an open access article distributed under the conditions of the Creative Commons by Attribution License, which permits unrestricted use, distribution, and reproduction in any medium or format, provided the original work is correctly cited. 
synergistic effects of yoga and exposure to nature is warranted to better understand whether the environment in which yoga is practiced could enhance the benefits of this mind-body modality on health.

\section{Keywords}

College students; green exercise; hatha yoga; indoor and outdoor environments; nature; PANAS; physiology

\section{Introduction}

College students are exposed to high stress levels that can affect their quality of life and academic performance. The World Mental Health International College Student Initiative (WMH-ICS) designed by the World Health Organization found that, of 20,842 students from 24 universities across 9 countries, $93.7 \%$ reported experiencing at least mild stress in one or more of the following categories: family relationships, financials, love life, personal health, problems of loved ones, and school/work relationships [1]. The WMH-ICS surveys also showed an association between extent of stress in each of those categories and increased chances of the college student having at least one of the following mental health conditions: attention deficit/hyperactivity disorder, bipolar I-II disorder, drug abuse or dependence, generalized anxiety disorder, major depressive disorder, and panic disorder [1]. Another survey from The American College Health Association (2019) revealed that $26 \%$ of students reported feelings of depression that impaired their functioning, and $43 \%$ of students reported feeling overwhelming anxiety over a period of 30 days [2]. Given the breath of information related to college life-induced stress and mental health [3], it is imperative to address this issue in early adulthood to alleviate associated acute symptoms as well as prevent chronic mental and physical health conditions that may persist or emerge later in life.

The practice of yoga is an exceptional method that can be used to relieve stress in college students. The term "yoga" means "yoke" or "union" and is a comprehensive system that originated in Ancient India about 5,000 years ago to balance the mind, body, and spirit for health enhancement $[4,5]$. There are many different types of yoga that are rooted in various contemplative traditions of Eastern philosophy and spiritual teachings [6]. Some styles of yoga incorporate practicing a series of physical postures, breathwork, and meditation techniques [7]. National surveys show that yoga practices are becoming more popular around the world [8]. However, yoga research in the United States about this ancient mind-body practice examining its potential as an integrative health and wellness modality is still in its infancy [9]. Specifically, more information is needed about the physiological and psychological benefits of yoga for college students.

The existing published research related to the college student population has mostly examined the impact of longitudinal yoga interventions. Such long-term studies suggest that yoga can positively impact body awareness, mental clarity, concentration, and energy [10], alleviate anxiety [11], decrease perceived stress levels [12], increase focus and feelings of empowerment [13], and improve mindfulness [14]. Additionally, one randomized controlled trial (RCT) found that college students who practiced 75-minutes of Ashtanga Vinyasa yoga twice a week for 12 weeks had less psychological distress and sleep problems post-intervention with effects sustained for three months 
[15]. In contrast, other studies investigating the effects of extended Hatha yoga interventions for nonclinical college students did not yield improvements in anxiety or stress, possibly due to methodological limitations such as a delay in the start of data collection, low baseline reports of the outcomes, and small samples [16, 17]. Thus far, the research cited describes findings where participants were involved in more than one yoga session over an extended period of time. Other studies that have investigated the acute psychological responses of college students to short-term yoga interventions revealed a significant amelioration of anxiety levels after a 30-minute yoga session [18, 19]. Moreover, 90-minutes of Hatha yoga significantly decreased negative affect [20] and 100-minutes of Vinyasa yoga significantly improved both positive and negative affect scores [21] in college student samples.

While there are several reasons to maintain a yoga practice, one of the most researched areas relates to the impact of yoga on physical health. Broadly, yoga has been found to relieve many symptoms of physical ailments in clinical adult populations [22] and to enhance cardiovascular health in a sample of nonclinical adults [23]. A recent meta-analysis found that mind-body interventions, including yoga, significantly decreased blood pressure, cortisol, and heart rate with effects tending to be stronger for students in secondary/higher education [24]. Yet, research about the physiological impacts of yoga for college students is scarce. One study suggested that college students who engaged in 90-minutes of yoga six times per week over five months showed improvements in heart rate variability [25]. Another long-term study found that college students who practiced 45-minute Hatha yoga sessions twice a week over a 16-week period showed a nonsignificant trend towards a reduction in blood pressure in comparison to a control group [26]. Research about the acute physiological responses of college students to a single yoga session is as scarce as the information previously referenced related to acute psychological outcomes. We found only one study in the literature showing that 30-minutes of restorative yoga significantly decreased blood pressure and heart rate for graduate students [27].

Another method that holds promising potential for enhancing college student health and wellness is exposure to natural environments. Humans evolved in close contact with nature. Yet, the rise of modern urban environments has resulted in a disconnection between humans and nature [28]. The Stress Reduction Theory suggests that exposure to certain natural environments may foster recovery from daily life stressors, and ultimately, enhance human health and well-being [29]. There is mounting evidence of the restorative effects that nature has on human physical and mental health [30]. Studies involving nature immersions have been shown to greatly improve biomarkers and self-reported stress in diverse human samples [31-33]. Likewise, studies completed in laboratory settings, such as viewing images and listening to sounds of nature, seem to be effective in relieving physiological stress [34, 35].

The term "green exercise" is particularly relevant to our study, since it is defined as "physical activity in green places that may bring both physical and mental health benefits" [36]. Exercise done in natural or "green" environments has been shown to improve self-esteem and mood while also fostering a sense of positive engagement and revitalization [37]. The most common type of green exercise study compares walking in natural versus urban environments [38, 39]. Other studies have also examined cycling [40], running [41], dancing [42], and practicing taekwondo [43] in natural and urban environments with middle-aged adult samples. However, to the best of our knowledge, no previous study has compared the effects of yoga sessions in outdoor versus indoor environments. 
The present study aims to contribute to emerging research areas that could benefit college students experiencing increased stress levels that may predispose them to long-term health complications. Our main goal was to compare the effects of yoga on physiological (i.e., heart rate, respiration rate, and perfusion index) and psychological (i.e., positive and negative affect) responses of college females when practiced in indoor and outdoor environments. After reviewing the literature about the positive implications of nature exposure and "green exercise" on human health [36], we hypothesized that the outdoor environment may act synergistically with the yoga practice to further decrease physiological stress responses (measured by heart rate and respiration rate), and negative affect while enhancing positive affect. Previous research has demonstrated that yoga is a useful method for enhancing both physical and psychological health over time, yet the acute benefits of a single Hatha yoga session for college students have been rarely reported. Therefore, a second objective of this study was to evaluate whether a single 40-minute session of Hatha yoga would be an efficient strategy to alleviate acute physiological stress responses and improve affect states of female college students. Lastly, the implications of these inquiries may be used to inform wellness protocols both as preventative and prescriptive initiatives to foster holistic health in a costeffective and accessible way for college students. The application of these initiatives would be important for this population since young adults are at a crucial life stage for the development of health management techniques.

\section{Materials and Methods}

The Bowling Green State University Institutional Review Board provided expedited review and approved the protocol for this study (project ID: 1419961-3; June 20, 2019).

\subsection{Subjects}

Participants were recruited using convenience sampling by email, flyers posted around campus, and via social media during summer 2019. Fifty-eight college females between the ages of 18-28 (mean $\pm \mathrm{SE}=21.6 \pm 0.37$ ) completed the study. Average resting heart rate is higher in females than males [44]; therefore, to account for potential differences in baseline physiological outcomes, we chose to only enroll female college students in this study. Other than demographics (age and sex), there were no additional exclusion criteria to participate in this study.

\subsection{Yoga Sessions}

Hatha yoga, the most practiced type of yoga within the context of Western healthcare [4], incorporates focused breathing, gentle stretching, and awareness of sensations to balance the mind and body [45]. This yoga style was taught to the participants and facilitated by the same certified yoga instructor (KMD) for all sessions. Specifically, the breakdown of the session included mindful breathing ( 3 minutes), yoga poses (32 minutes), and meditation (5 minutes). For the mindful breathing, participants were instructed to focus attention to their natural breathing before following a pattern led by the yoga instructor that included inhaling for a count of 4 , holding the breath for a count of 4 , and exhaling for a count of 6 . The yoga poses included: Bridge (Setu Bandha Sarvāñgāsan), Cradle the Baby (Hindolasana), Table Top (Bharmanasana), Cat (Mārjarīāsana), Cow (Bitilāsana), Child's Pose (Bālāsana), Downward Facing Dog (Adho Mukha Śvānāsana), Mountain 
(Tāọāsana), Warrior I (Virrabhadrāsana I), Warrior II (Vïrabhadrāsana II), Triangle (Utthita Trikoṇāsana), Tree (Ṿ̣kṣāsana), Forward Fold (Uttānāsana), Butterfly (Baddhakoṇāsana), Seated Straddle Split (Samakonasana), Staff (Daṇ̣̂āsana), and Boat (Naukāsana). While the order of yoga postures may vary depending on the modality of the yoga practice and tradition, the postures in this study were chosen to include a variety of movements that would include the three planes of seated, standing, and supine with simple physical transitions. For the closing meditation, participants rested on their backs and were encouraged to follow the natural rhythm of their breath without any guiding words from the yoga instructor to practice mindfulness in the context of breath attention. Further, there were no guiding words from the instructor to foster the possibility for participants to be fully aware of the possible sounds present within the environment they were assigned to practice the yoga session.

\subsection{Indoor and Outdoor Environments}

The participants were randomly assigned to complete a 40-minute session of Hatha yoga taking place either in an indoor (i.e., an empty classroom; $N=30$ ) or outdoor (i.e., in a park; $N=28$ ) environment located on the Bowling Green State University campus (Figure 1). All the yoga sessions took place from 8-9 pm on Monday through Thursday and occurred from 10 July until 22 August 2019. The number of participants in each yoga session ranged from 2-9. Each participant received a new yoga mat to use during the study and to keep afterward. The yoga mats were arranged in a semi-circle facing the yoga instructor in the center to ensure that each participant had an unobstructed view of the instructor in both environments. The indoor participants faced a bare classroom wall without any windows to nature, and the fluorescent lights were turned off for the duration of the yoga session. The outdoor participants placed their yoga mats on grass, were surrounded by large trees, and faced a small garden with flowers, shrubs, and other trees.

$\mathbf{a}$
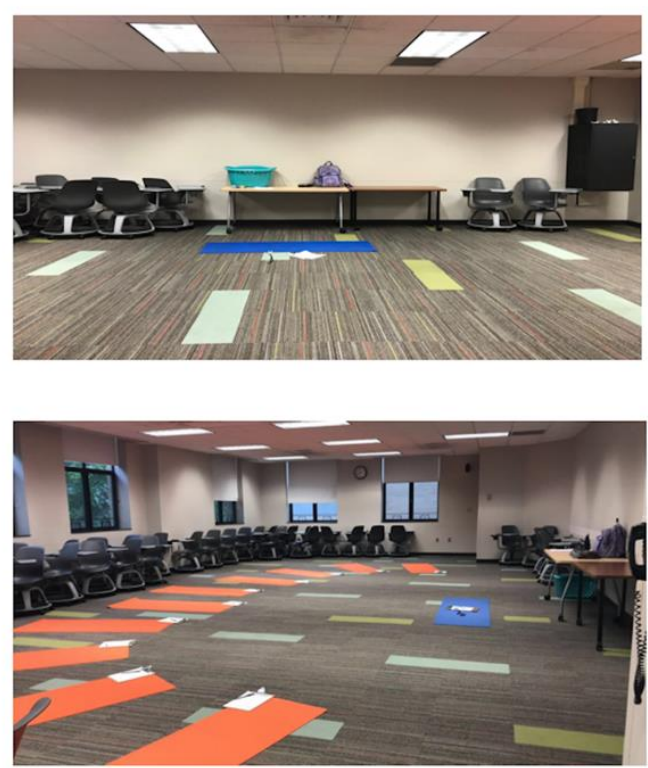

b
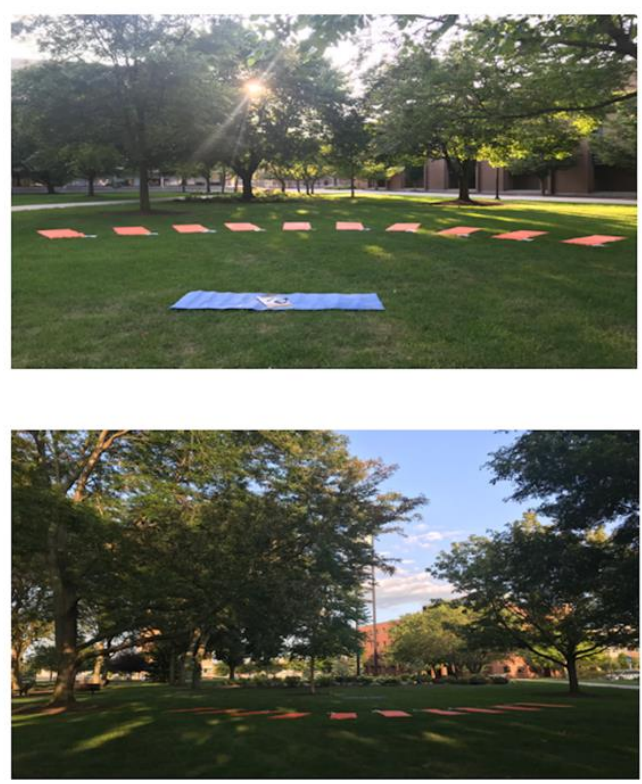

Figure 1 Photographs of the two environments for this study: (a) Eppler South 307 classroom (indoor environment), and (b) Carillon Park (outdoor environment). Both environments were located on the Bowling Green State University campus. 


\subsection{Experimental Protocol}

Each participant was provided a clipboard with a packet of necessary documents to be used during the study. Written consent was obtained from each participant before the start of the yoga session. First, the participants completed the Positive and Negative Affect Schedule (PANAS) [46]. This questionnaire consists of 20 affect states that the participants were asked to rate using a 5point Likert Scale ranging from 1 (very slightly or not at all) to 5 (extremely) based on how they were feeling at the time. The 10-items in the PANAS related to positive affect (PA) include: active, alert, attentive, determined, enthusiastic, excited, inspired, interested, proud, and strong. The 10-items associated with negative affect (NA) include: afraid, ashamed, distressed, guilty, hostile, irritable, jittery, nervous, scared, and upset. The total scores for the PA and NA were calculated separately by adding the individual responses for all items within each affect category. These total scores could range from 10 to 50 with higher scores representing higher self-reported affect. Good internal consistency and adequate test-retest reliability correlations for both the PA and NA scales have been reported [46].

After the PANAS was completed by the participants, respiration rate (RR) and manual heart rate (HR) were recorded as a group. To record RR, the participants were instructed to count the number of times that they inhaled (i.e., every time the chest/abdomen rises) during a 60-second period. The participants were notified when to begin and cease counting, and then to record the number of breaths on a card provided to them. Next, to record manual heart rate (HR), the participants were instructed to place their index and middle fingers to the side of their neck on the windpipe to find their carotid pulse. The participants were instructed when to begin counting and when to stop after 15-seconds, and to record the HR on their data card. Finally, to measure perfusion index (PI; indicator of pulse strength) and a second measure of HR, a pulse oximeter was used. The device was clipped to the tip of the right index finger of each participant individually for a few seconds, and the readings were recorded by one of the researchers. The measures of $R R$, manual $H R$, and pulse oximeter HR and PI were the selected physiological variables for this study due to the ability to quickly record these biomarkers of stress individually within a group setting. After recording baseline data, the participants completed the 40-minute yoga session, followed by the same procedure for data collection in the following order: RR, manual HR, pulse oximeter HR and PI. Next, the participants answered the PANAS questionnaire to rate each item based on how they were feeling immediately after the yoga session. Lastly, participants completed some brief questions about their experience with the yoga session and the environment they were randomly assigned to (either indoor or outdoor). The total time commitment for the study was 60 minutes.

\subsection{Statistical Analyses}

Repeated measures ANOVAs were performed to evaluate the effects of the yoga session (baseline and after yoga measurements), environment (indoor and outdoor), and the interaction of the yoga session and the environment on vital signs (i.e., respiration rate, heart rate measures, and perfusion index) as well as on PANAS scores. Data was rank transformed for total PA and NA scores (non-parametric tests), and also for any other variable that deviated from ANOVA assumptions of normality and homogeneity of variance. Pairwise comparisons among treatment means were performed using Fisher's Least Significant Difference tests only when ANOVA models were 
significant (protected LSD). Statistical analyses were performed using SAS version 9.4 (SAS Institute, Cary, NC, USA).

\section{Results}

Analyses of variance indicated that the yoga session had significant effects on manual heart rate and respiration rate (Table 1; $\mathrm{P}<0.01$ ). These two measurements significantly decreased after yoga in both the indoor and outdoor groups (Figure $2 \mathrm{a}-\mathrm{b}$ ). However, neither the yoga session or the environment significantly affected heart rate and perfusion index recorded with the pulse oximeter (Figure $2 \mathrm{c}$-d). The ANOVA also revealed that the yoga practice significantly influenced negative affect scores (Table 1; $P<0.01$ ), as shown by the decreased NA values after the session in both environments (Figure $3 \mathrm{~b}$ ). The environment per se also significantly influenced negative affect scores (Table 1). There were lower negative affect baseline levels in the outdoor compared to the indoor environment (Figure $3 \mathrm{~b}$ ). In addition, while there was a trend toward increases in positive affect scores at the completion of the yoga sessions in both environments, the results were not significant (Figure $3 \mathrm{~b}$ ). Lastly, the effect of yoga by environment interactions were not significant for any of the variables indicating that the yoga session influenced physiological and psychological variables in the same way regardless of the environment in which it was practiced.

Table 1 Analyses of variance evaluating the effects of the yoga session and the environment (indoor and outdoor) on physiological and psychological variables.

\begin{tabular}{|c|c|c|c|c|}
\hline & & Yoga & Environment & Yoga $\times$ Environment \\
\hline & DF & F-values & & \\
\hline Heart Rate (Manual) & 1,112 & $10.23^{*}$ & 0.98 & 0.77 \\
\hline Heart Rate (Oximeter) & 1,112 & 1.29 & 3.53 & 0.50 \\
\hline Perfusion Index & 1,112 & 0.39 & 0.17 & 0.42 \\
\hline Respiration & 1,112 & $19.24 *$ & 0.05 & 0.35 \\
\hline Positive Affect & 1,112 & 3.08 & 0.18 & 0.80 \\
\hline Negative Affect & 1,112 & $28.59 *$ & $7.03^{*}$ & 0.67 \\
\hline
\end{tabular}

$$
* P<0.01
$$


$\mathbf{a}$

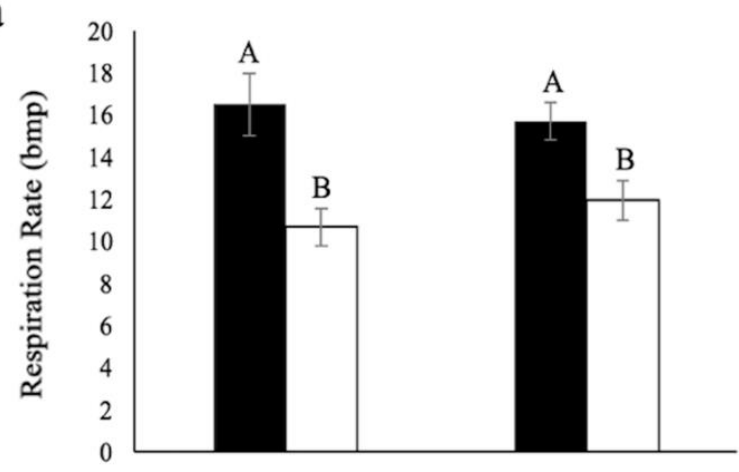

c

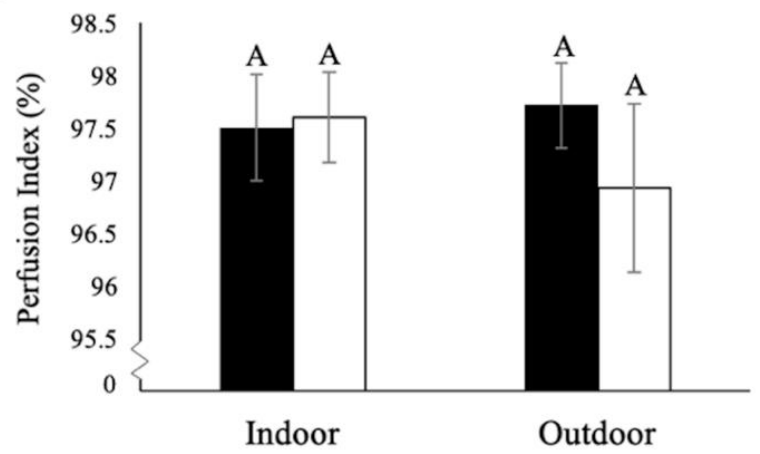

b

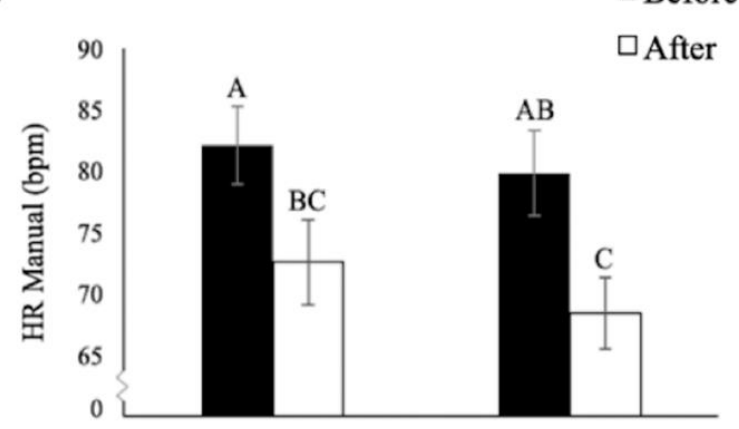

d

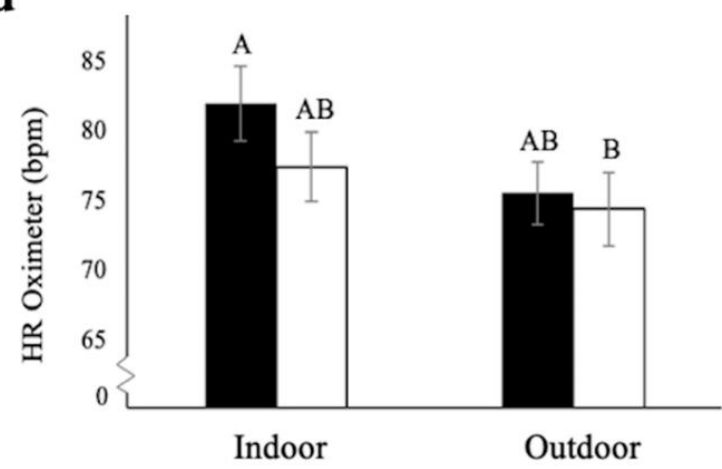

Figure 2 Effects of the yoga session (before and after) and environment (indoor and outdoor) on means ( \pm standard errors) of the following vital signs: a) respiration rate (breaths per minute), b) heart rate (HR) measured manually, c) perfusion index, and d) heart rate $(H R)$ measured with a pulse oximeter. Different letters denote a statistically significant difference between the means of the measured variables $(P<0.05)$. 


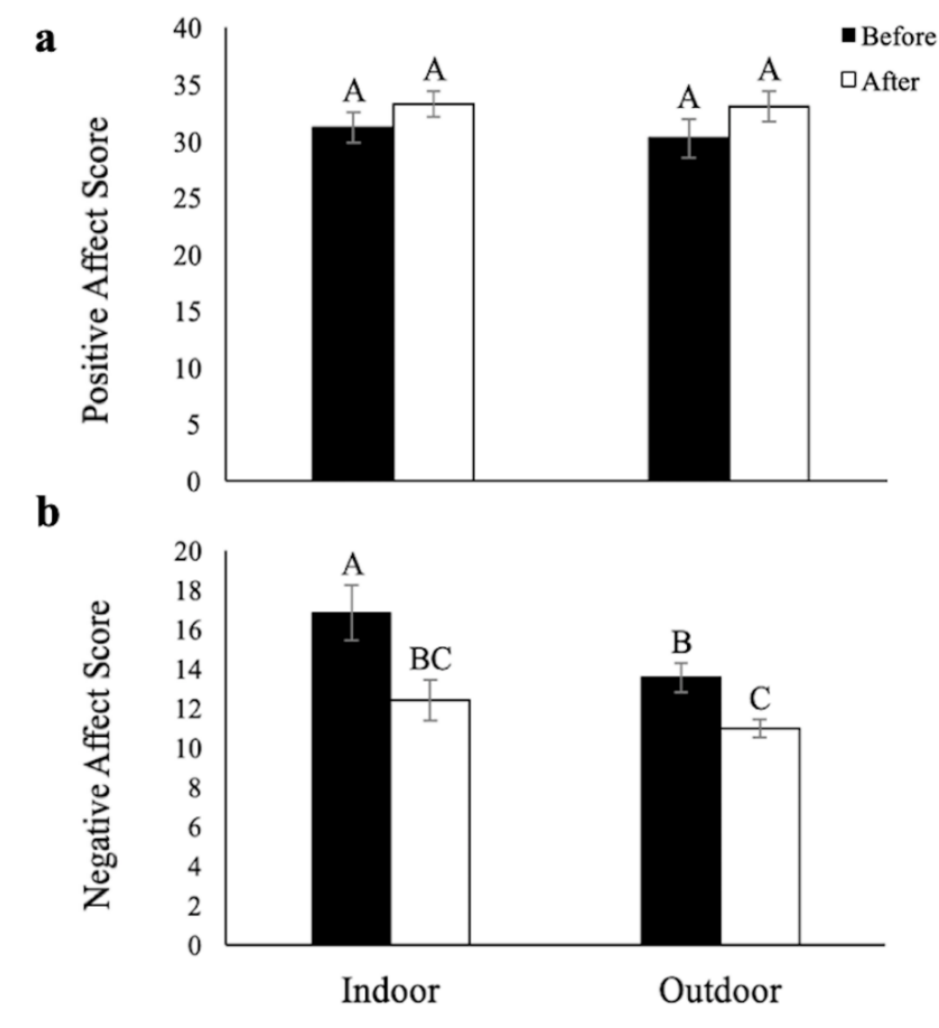

Figure 3 Effects of the yoga session (before and after) and the environment (indoor and outdoor) on the Positive and Negative Affect Schedule (PANAS) questionnaire scores. Means and standard error bars for positive (a) and negative (b) affect scores. Different letters denote a statistically significant difference between the means of the measured variables $(P<0.05)$.

Information from some general questions revealed that 24 out of the total 58 participants never practiced yoga before and novice yoga practitioners were balanced between the two groups (indoor versus outdoor). When asked whether they would participate in another yoga session located in the same environment, most participants indicated that they would take another yoga class in the environment they were randomly assigned to (i.e., $90 \%$ and $100 \%$ positive responses for participants assigned to the indoor and outdoor groups, respectively). Participants were also asked to identify their favorite component of the yoga class from five options. Order of preferences for these components reported by the indoor group were deep breathing (37\%), meditation (30\%), stretching (30\%), and postures (3\%). No participants from the indoor group chose "location" as their favorite component of the yoga session. Component rankings reported by the outdoor group included location (36\%), meditation (21\%), stretching (21\%), postures (14\%), and deep breathing (8\%). In the outdoor group, "location" was the most-reported favorite component of the yoga session.

\section{Discussion}

Results from this study suggest that 40-minutes of Hatha yoga had some benefits for college females. Self-reported measures of respiration rate and heart rate significantly decreased after the 
yoga sessions in both environments and may indicate alleviation of acute physiological stress. However, the significant decrease in heart rate was detected only when this variable was measured individually by each participant immediately after the yoga practice ended in contrast to the second heart rate measure using a pulse oximeter. The lack of significant results for the second HR measure could have resulted from the time lapse that occurred as participants had to wait for the researcher to record heart rate using the pulse oximeter one participant at a time. This could have also introduced some stress on the participants. Similarly, there were no significant differences in perfusion index that was also measured via the pulse oximeter.

Additionally, our study demonstrated that negative affect significantly decreased after 40minutes of Hatha yoga in both environments. In contrast, while there were slight increases in positive affect for both environments, the results were not significant. These outcomes mirror another study that found significant decreases in negative affect, but no significant change in positive affect, for college students who completed a 90-minute Hatha yoga class [20]. The lack of significant increase in positive affect could be related to high baseline levels of positive affect in students that participated in this free yoga class during a summer season. Greater baseline positive affect levels may also be due to the increasing utilization and acceptance of yogic techniques for health enhancement. Mindfulness-based movement modalities for personal wellness are increasing in popularity with yoga being the method most widely used in the United States [47]. Our results related to yoga-induced decreases in negative affect were also in accordance with those of a previous study where college students showed significant reductions in negative affect after most sessions as well as significant improvements in positive affect for fourteen out of sixteen sessions [21]. Like in our study, these researchers also found that a single yoga session may induce a significant decrease in negative affect scores of college students. Interestingly, the Hatha yoga classes in our study lasted 40 minutes while the aforementioned study [21] included Vinyasa yoga classes for 100 minutes. Thus, there could be mixed results regarding the acute impacts of different types of yoga on positive and negative affect in college student samples. Future research with more robust methodological approaches, such as including a control group, should be considered as well as the style of yoga and duration of the practice session.

Overall, neurophysiological and neurobiochemical changes reported after the practice of yoga deserve further evaluation, especially those related to the amelioration of stress. A review of yoga studies with non-clinical samples found that yogic techniques positively impacted brain wave activity and amygdala activation; these impacts have been associated with improvements in mood and anxiety [48]. Additionally, one counterbalanced, randomized-crossover trial found that 30minutes of Hatha yoga significantly ameliorated salivary cortisol levels in young adults after an acute stress task compared to the control condition (watching TV) [49]. Another study also found that 30minutes of Hatha yoga significantly reduced salivary cortisol levels for young adults whereas a timematched trial of power yoga did not affect [19]. The type of yoga practice may be an important consideration for designing studies as the variability in yoga styles might induce opposing effects for participants. In contrast, while a trend towards lower cortisol levels has been shown in adults with two years of yoga experience, the results were not significantly lower when compared to individuals who did not practice yoga [50]. These outcomes are important to better understand what type of yoga would be most beneficial based on different contexts, characteristics, and goals of the practitioners. Therefore, it may be worthwhile to incorporate markers related to neurophysiology 
and biochemistry to yoga research to further assess the multidisciplinary role of this mind-body practice on health-related outcomes.

A second main objective of our study was to compare the impacts of single Hatha yoga sessions occurring in outdoor versus indoor environments. Baseline reports of negative affect were significantly lower in students assigned to the outdoor yoga session compared to those assigned to the indoor location (a commonly used college classroom). One possible explanation for this result could have been due to the students' previous perceptions of college classrooms being associated with negative states, such as stress and anxiety, when attending courses or taking exams. However, there were no significant interaction effects between yoga and the environment indicating that effects of the yoga session were not influenced by the location in which it was practiced. This finding is similar to previous research that also did not find significant differences in acute psychological constructs between exercise sessions completed in outdoor and indoor locations [51]. Furthermore, the participants in our study completed a general questionnaire about the yoga session. Twentyfour out of the total 58 participants never practiced yoga before. This may denote an interest of college females for trying this mind-body practice and could possibly support efforts by universities to incorporate yoga in wellness initiatives. Regarding environmental effects, some of the participants reported that they were uncomfortable practicing yoga outside due to the texture of the grass, prevalence of insects, loud sound of cicadas, and humidity. These elements of nature may be unfamiliar and even stress-inducing especially for people that live or were raised in urban areas. Reconnection with nature via regular exposure to this environment may alleviate potential perceptions of discomfort. Despite these observations, all the participants who completed the outdoor yoga session reported they would still take another yoga session outdoors in the future. In addition, over $30 \%$ of the outdoor participants chose location as their favorite component of the yoga session over the other choices of deep breathing, meditation, postures, and stretching. Environment preference may be an important factor to consider when planning yoga sessions for college students to possibly enhance initial interest and maintain motivation to practice. For example, one study reported that natural environments were rated by exercisers as top venues of choice due to their convenience [52]. The outdoor yoga sessions in our study took place in a park located on a public college campus, where students may choose to personally practice mind-body techniques, such as yoga, and where group classes could easily be held.

This study showed that yoga can have positive effects on physiological and psychological responses of college females. Some ways in which this type of study can be improved in the future is by adding a control group (i.e., indoor and outdoor groups not practicing yoga) to more accurately test for the effects of yoga sessions on the response variables. Secondly, the outdoor environment was located on a public college campus and could not be controlled by the researchers. Using outdoor spaces for exercise always harbors the potential that other people and activities may be present in the surrounding area. In the sessions for this study, there were no major disruptions most of the time; however, there was occasionally someone walking by that could have caused some distraction or discomfort for the participants. Also, some of the outdoor classes that occurred later in August had the impact of artificial light due to the sun setting earlier, which initiated the automatic campus light posts to turn on. Thirdly, the generalizability of these results is limited to female college students between the ages of 18-28. A sampling bias may be present as females could possibly be more interested in participating in a yoga study. There is a known higher female to male ratio related to the practice of yoga in Western societies [53]. Another limitation could have 
been due to biases related to self-measured RR and the first HR variable. To address this concern, a pulse oximeter was used to record a second measure of $H R$, yet this method was also limited in that there was a time lag between the conclusion of the yoga session and recording this measure for each participant. Future studies may consider providing each participant with their own pulse oximeter to obtain a more immediate and accurate post-yoga outcome. Lastly, a health screening process was not used. Future studies may want to incorporate these measures for a more detailed understanding of the potential influences of yoga practiced in different environments on markers related to health.

Despite some limitations, our study is unique for several reasons. To our knowledge, no study has examined the effects of yoga in natural environments or compared the impacts of yoga practiced in different locations (i.e., indoor versus outdoor). Other types of "green exercise" [36] have been studied, but not as frequently with college students, a population at-risk of developing stress-related health conditions. College student health may be improved by engaging in outdoor physical activity while living or attending classes on campus. Location might be an important variable to consider for yoga classes, especially if the outdoor environment can enhance the benefits of this mind-body practice. Our study found limited differences between environments (only for baseline negative affect); however, this is a topic that deserves further investigation. For example, college students actively involved in physical activities (i.e., biking, running, and walking) in green spaces noted greater life quality, enhanced mood, and less stress compared to those engaged in more passive activities, such as sitting, studying, relaxing with friends, and meditating in the same outdoor environment [54]. Therefore, the type of activity taking place in an outdoor environment is also a variable to consider when examining the potential influences of green spaces on college student health outcomes. Depending on accessibility, exposure to natural environments may provide holistic health benefits that are cost effective, pose low risk for college students, and may improve their psychophysiological responses to stress.

As previously noted, there are very few studies that have aimed at examining the acute effects of single Hatha yoga classes, as presented here. Single sessions of yoga may be most feasible for college students who are very busy and challenged to establish wellness regiments while learning how to balance the constant stressors associated with college life. While a regular yoga practice would be more effective for maintaining stress relief and positive health outcomes, college students may still benefit from the acute effects of single yoga classes at times when they are experiencing high stress. Yoga initiatives may be integrated on college campuses within college counseling centers [55] and through offering classes at the student recreation center, courses available for university credit, or student organizations. Future studies are recommended to indicate the immediate benefits of yoga practice because this information may be useful for motivating college students and universities to utilize yoga as a possible wellness initiative if there is a welldocumented evidence base of health enhancing outcomes.

\section{Conclusions}

The results from our study suggest that 40-minutes of a single Hatha yoga session can be useful to improve physiological markers of stress, such as decreasing self-reported heart rate and respiration rate, as well as ameliorating negative affect states for college females. These results were found regardless of the environment in which yoga was practiced, although the environment 
seemed to influence baseline negative affect. Further research is needed to gather more information on how both Hatha yoga and the environment in which it is practiced may influence the psychophysiology of college students.

\section{Acknowledgments}

We are thankful for the college students that completed the yoga sessions, and Bowling Green State University for funding this study.

\section{Author Contributions}

Both authors designed this study and worked together to write this manuscript. KMD taught the yoga sessions and MGB performed the statistical analyses.

\section{Funding}

This study was financially supported by the BGSU Center for Undergraduate Research and Scholarship.

\section{Competing Interests}

The authors have declared that no competing interests exist.

\section{References}

1. Karyotaki E, Cuijpers P, Albor Y, Alonso J, Auerbach RP, Bantjes J, et al. Sources of stress and their associations with mental disorders among college students: Results of the world health organization world mental health surveys international college student initiative. Front Psychol. 2020; 11: 1759.

2. American college health association. American college health association-national college health assessment II: Reference group executive summary spring 2019. Silver Spring MD: American college health association; 2019; 2.

3. Lui CH, Stevens C, Wong SH, Yasui M, Chen JA. The prevalence and predictors of mental health diagnoses and suicide among U.S. college students: Implications for addressing disparities in service use. Depress Anxiety. 2019; 36: 8-17.

4. Salmon $P$, Lush E, Jablonski M, Sephton SE. Yoga and mindfulness: Clinical aspects of an ancient mind/body practice. Cogn Behav Pract. 2009; 16: 59-72.

5. Gard T, Noggle JJ, Park CL, Vago DR, Wilson A. Potential self-regulatory mechanisms of yoga for psychological health. Front Hum Neurosci. 2014; 8: 770.

6. Feuerstein $\mathrm{G}$. The yoga tradition: Its history, literature, philosophy and practice. 3rd ed. Chino Valley: Hohm Press; 1998.

7. Ariel-Donges AH, Gordon EL, Bauman V, Perri MG. Does yoga help college-aged women and body-image dissatisfaction feel better about their bodies? Sex Roles. 2019; 80: 41-51.

8. Park CL, Riley KE, Bedesin E, Stewart VM. Why practice yoga? Practitioners' motivations for adopting and maintaining yoga practice. J Health Psychol. 2016; 21: 887-896.

9. Michelis ED. A preliminary survey of modern yoga studies. Asian Med. 2007; 3: 1-19. 
10. Schure MB, Christopher J, Christopher S. Mind-Body medicine and the art of self-care: Teaching mindfulness to counseling students through yoga, meditation, and qigong. J Couns Dev. 2008; 86: 47-56.

11. Yüce GE, Muz G. Effect of yoga-based physical activity on perceived stress, anxiety, and quality of life in young adults. Perspect Psychiatr Care. 2020; 56: 697-704.

12. SchmalzI L, Powers C, Zanesco AP, Yetz N, Groessl EJ, Saron CD. The effect of movement-based and breath-focused yoga practice on stress parameters and sustained attention: A randomized controlled pilot study. Conscious Cogn. 2018; 65: 109-125.

13. Villate VM. Yoga for college students: An empowering form of movement and connection. Phys Educ. 2015; 72: 44-66.

14. Lemay V, Hoolahan J, Buchanan A. Impact of a yoga and meditation intervention on students' stress and anxiety levels. Am J Pharm Educ. 2019; 83: 747-752.

15. Elstad T, Ulleberg P, Klonteig S, Hisdal J, Dyrdal GM, Bjorndal A. The effects of yoga on student mental health: A randomized controlled trial. Health Psychol Behav Med. 2020; 8: 573-586.

16. Cohrs JY, Throne MB. The effects of yoga on the psychological health of college students. Gainesville: Brenau University; 2016.

17. Papp ME, Nygren-Bonnier M, Gullstrand L, Wandell PE, Lindfors P. A randomized controlled pilot study of the effects of 6-week high intensity hatha yoga protocol on health-related outcomes among students. J Bodyw Mov Ther. 2019; 23: 766-772.

18. Albracht-Schulte K, Robert-McComb J. The effects of yoga and quiet rest on subjective levels of anxiety and physiological correlates: A 2-way crossover randomized trial. BMC Complement Altern Med. 2018; 18: 280.

19. Marshall M, McClanahan M, Warren SM, Rogers R, Ballmann C. A comparison of the acute effects of different forms of yoga on physiological and psychological stress: A pilot study. Int J Environ Res Public Health. 2020; 17: 6090.

20. West J, Otte C, Geher K, Johnson J, Mohr DC. Effects of hatha yoga and African dance on perceived stress, affect, and salivary cortisol. Ann Behav Med. 2004; 28: 114-118.

21. Gaskins $R$, Jennings $E$, Thind $H$, Becker B, Bock B. Acute and cumulative effects of vinyasa yoga on affect and stress among college students participating in an eight-week yoga program: $A$ pilot study. Int J Yoga Therap. 2014; 24: 63-70.

22. Field T. Yoga research review. Complement Ther Clin Pract. 2016; 24: 145-161.

23. Satin JR, Linden W, Millman RD. Yoga and psychophysiological determinants of cardiovascular health: Comparing yoga practitioners, runners, and sedentary individuals. Ann Behav Med. 2014; 47: 231-241.

24. Strehli I, Burns RD, Bai Y, Ziegenfuss DH, Block ME, Brusseau TA. Mind-body physical activity interventions and stress-related physiological markers in educational settings: A systemic review and meta-analysis. Int J Environ Res Public Health. 2021; 18: 224.

25. Nagendra H, Kumar V, Mukherjee S. Cognitive behavior evaluation based on physiological parameters among young healthy subjects with yoga as intervention. Comput Math Methods Med. 2015; 2015: 821061.

26. Nelson D, Reed J, Buck SM. Effect of a 16-week yoga program on blood pressure in healthy college students. Phys Educ. 2014; 71: 533-544.

27. Rizzolo D, Zipp GP, Stiskal D, Simpkins S. Stress management strategies for students: The immediate effects of yoga, humor, and reading on stress. J Coll Teach Learn. 2009; 6: 79-88. 
28. Maller C, Townsend M, Pryor A, Brown P, Leger L. Healthy nature healthy people: "Contact with nature" as an upstream health promotion intervention for populations. Health Promot Int. 2006; 21: 45-54.

29. Ulrich RS. Aesthetic and affective response to natural environment. In: Human behavior and environment. Boston MA: Behavior and natural environment; 1983. pp 85-125.

30. Bratman GN, Hamilton JP, Daily GC. The impacts of nature experience on human cognitive function and mental health. Ann N Y Acad Sci. 2012; 1249: 118-136.

31. Song $\mathrm{C}$, Ikei $\mathrm{H}$, Miyazaki Y. Physiological effects of nature therapy: A review of the research in Japan. Int J Environ Res Public Health. 2016; 13: 781.

32. Hansen MM, Jones R, Tocchini K. Shinrin-yoku (forest bathing) and nature therapy: A state-ofthe-art review. Int J Environ Res Public Health. 2017; 14: 851.

33. Kondo MC, Jacoby SF, South EC. Does spending time outdoors reduce stress? A review of realtime stress response to outdoor environments. Health Place. 2018; 51: 136-150.

34. Gladwell V, Brown DK, Barton J, Tarvainen MP, Kuoppa P, Pretty JN, et al. The effects of views of nature on automatic control. Eur J Appl Physiol. 2012; 112: 3379-3386.

35. Ulrich RS, Simons RF, Losito BD, Fiorito E, Miles MA, Zelson M. Stress recovery during exposure to natural and urban environments. J Environ Psychol. 1991; 11: 201-230.

36. Pretty JN, Griffin M, Sellens M, Pretty C. Green exercise: Complementary roles of nature, exercise and diet in physical and emotional well-being and implications for public health policy. Colchester: University of Essex; 2003.

37. Gladwell VF, Brown DK, Wood C, Sandercock GR, Barton JL. The great outdoors: How a green exercise environment can benefit all. Extreme Physiol Med. 2013; 2: 3.

38. de Brito JN, Pope ZC, Mitchell NR, Schneider IE, Larson JM, Horton TH, et al. Changes in psychological and cognitive outcomes after green versus suburban walking: A pilot crossover study. Int J Environ Res Public Health. 2019; 16: 2894.

39. Koselka E, Weidner LC, Minasov A, Berman MG, Leonard WR, Santoso MV, et al. Walking green: Developing an evidence base for nature prescriptions. Int J Environ Res Public Health. 2019; 16: 4338.

40. Rogerson M, Gladwell VF, Gallagher DJ, Barton JL. Influences of green outdoors versus indoors environmental settings on psychological and social outcomes of controlled exercise. Int J Environ Res Public Health. 2016; 13: 363.

41. Turner TL, Stevinson C. Affective outcomes during and after high-intensity exercise in outdoor green and indoor gym settings. Int J Environ Health Res. 2017; 27: 106-116.

42. Byrka K, Ryczko N. Positive effects of dancing in natural versus indoor settings: The mediating role of engagement in physical activity. J Environ Psychol. 2018; 57: 25-33.

43. Jang $\mathrm{S}$, So $\mathrm{W}$. The effect of short-term outdoor taekwondo training on the concentration and mood of taekwondo players. World J Men's Health. 2017; 13: e68-e75.

44. Prabhavathi K, Selvi KT, Poornima KN, Sarvanan A. Role of biological sex in normal cardiac function and in its disease outcome-A review. J Clin Diagn Res. 2014; 8: be01-be04.

45. Kabat-Zinn J, Hanh TN. Full catastrophe living: Using the wisdom of your body and mind to face stress, pain and illness. 2nd ed. New York: Bantam Books; 2013.

46. Watson D, Clark LA, Tellegen A. Development and validation of brief measures of positive and negative affect: The PANAS scales. J Pers Soc Psychol. 1998; 54: 1063-1070. 
47. Clarke TC, Black LI, Stussman BJ, Barnes PM, Nahin RL. Trends in the use of complementary health approaches among adults: United States, 2002-2012. Natl Health Stat Report. 2015: 79.

48. Desai R, Tailor A, Bhatt T. Effects of yoga on brain waves and structural activation: A review. Complement Ther Clin Pract. 2015; 21: 112-118.

49. Benvenutti MJ, Alves E, Michael S, Ding D, Stamatakis E, Edwards KM. A single session of hatha yoga improves stress reactivity and recovery after an acute psychological stress task-A counterbalanced, randomized-crossover trial in healthy individuals. Complement Ther Med. 2017; 35: 120-126.

50. Yoshihara K, Hiramoto T, Sudo N, Kubo C. Profile of mood states and stress-related biochemical indices in long-term yoga practitioners. Biopsychosoc Med. 2011; 5: 6.

51. Klaperski S, Koch E, Hewel D, Schempp A, Müller J. Optimizing mental health benefits of exercise: The influence of the exercise environment on acute stress levels and wellbeing. Ment Health Prev. 2019; 15: 200173.

52. Calogiuri G, Elliot LR. Why do people exercise in natural environments? Norwegian adults' motives for nature-, gym-, and sports-based exercise. Int J Environ Res Public Health. 2017; 14: 377.

53. Ross A, Friedmann E, Bevans $M$, Thomas S. National survey of yoga practitioners: Mental and physical health benefits. Complement Ther Med. 2013; 21: 313-323.

54. Holt EW, Lombard QK, Best N, Smiley-Smith S, Quinn JE. Active and passive use of green space, health, and well-being amongst university students. Int J Environ Res Public Health. 2019; 16: 424.

55. Adams CM, Puig A. Incorporating yoga into college counselling. J Creat Ment Health. 2008; 3: 357-372.

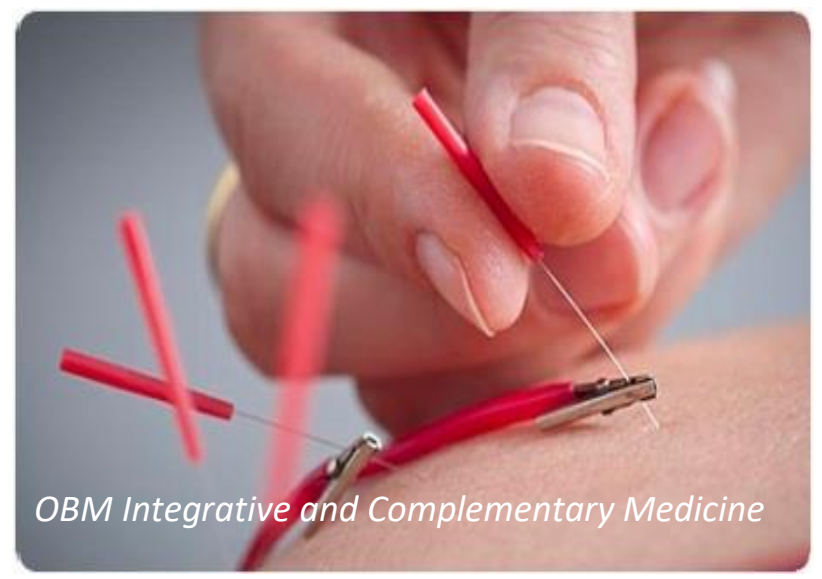

Enjoy OBM Integrative and Complementary Medicine by:

1. Submitting a manuscript

2. Joining in volunteer reviewer bank

3. Joining Editorial Board

4. Guest editing a special issue

For more details, please visit: http://www.lidsen.com/journals/icm 